\title{
Short-term weight-mediated effects of sleeve gastrectomy on echocardiographic surrogate markers of atherosclerotic vascular disease
}

Giovanni Casella, Lidia Castagneto-Gissey

Department of Surgical Sciences, "Sapienza" University of Rome, Italy

RELATED ARTICLE

by Kaya and Elkan, see p. 674

Correspondence to:

Giovanni Casella, MD, PhD, Department of Surgical Sciences, "Sapienza" University of Rome, Viale Regina Elena 324, 00161 Rome, Italy, phone: +390649975515, email: giovanni.casella@uniroma1.it Received: May 6, 2020. Accepted: May 7, 2020. Published online: August 25, 2020. Kardiol Pol. 2020; 78 (7-8): 655-656 doi:10.33963/KP.15560 Copyright by the Author(s), 2020
Overweight and obesity have reached pandemic proportions worldwide, affecting 39\% and 13\% of the adult population, respectively. ${ }^{1}$ Obesity is associated with the development of an array of metabolic complications, including insulin resistance, type 2 diabetes, hypertension, and dyslipidemia, which are correlated with an increased overall cardiovascular risk. Furthermore, obesity is regarded as an independent risk factor for cardiovascular disease (CVD), which may endure additive effects with the coexisting obesity-related disturbances, translating into the main cause of reduced overall survival rates in such individuals. ${ }^{2}$

Bariatric and metabolic surgery (BMS) has been extensively demonstrated to be the only effective treatment modality to date able to generate substantial and durable weight loss and resolution or improvement of comorbidities. ${ }^{3}$ Bariatric and metabolic surgery has been also specifically shown to decrease cardiovascular events in the long term as well as the associated morbiditiy and mortality, altogether reducing the related economic burden. ${ }^{4}$ Laparoscopic sleeve gastrectomy (LSG) is by all means the most commonly executed bariatric operation at present due to its proven effectiveness in terms of both weight and metabolic outcomes, deemed superimposable to those of other long-standing bariatric procedures such as Roux-en-Y gastric bypass. ${ }^{3}$ Moreover, LSG is now undoubtedly recognized as a metabolic procedure, and not as a merely restrictive one, since it is endowed with the capacity to induce the resolution of obesity-related metabolic complications-especially insulin resistance-before any substantial weight loss has been reached. ${ }^{5,6}$
In this issue of Kardiologia Polska (Kardiol Pol, Polish Heart Journal), Kaya et al ${ }^{7}$ illustrated the results of their prospective study including $71 \mathrm{pa}-$ tients affected by morbid obesity undergoing LSG, with the aim of analyzing the effects of this bariatric procedure on atherosclerotic vascular disease. To do so, authors specifically assessed multiple surrogate markers commonly used to evaluate the atherosclerotic burden, namely, aortic propagation velocity (APV), carotid intima-media thickness (CIMT), ankle-brachial index (ABI), and epicardial fat thickness. Emerging data from this study highlighted a significant reduction of body weight, additionally to a substantial improvement in all examined atherosclerotic markers 6 months after LSG. In particular, authors found a significant increase in APV and $\mathrm{ABI}$, together with a reduction of CIMT, epicardial fat thickness, systolic and diastolic blood pressure, triglyceride and low-density lipoprotein cholesterol levels. These results were strongly correlated with the degree of weight loss (ie, $\Delta$ body mass index). Such correlation allows to infer that the beneficial effects of LSG on atherosclerotic vascular disease markers are largely mediated by the entity of weight reduction obtained through surgery. In fact, in this regard, the authors assert that body mass index is a fundamental predictor for both changes in APV and CIMT.

A limited number of studies have described the positive long-term effects of different types of BMS procedures on reverse cardiac remodeling and cardiovascular events which occur consequently to considerable body weight reduction. ${ }^{8-10}$ In particular, our group previously analyzed in a retrospective study the echocardiographic and metabolic changes occurring 16 months after 
LSG. This study highlighted the beneficial modifications of the left ventricular shape in terms of mass, geometry, and diastolic function, altogether reducing left ventricular hypertrophy, which often develops in obese subjects. Interestingly, such results correlated not only with the degree of weight loss, but also with the remission of type 2 diabetes, obstructive sleep apnea, hypertension, and dyslipidemia. ${ }^{10}$ We further investigated, in another cohort of patients, the impact of LSG on cardiac function and metabolomic profile. This prospective study allowed us to confirm the significant echocardiographic amelioration after LSG and its stability over the 4-year follow-up period (Casella G et al, unpublished data, 2020). Furthermore, the metabolomic profile demonstrated to be a clinically valuable predictive factor of treatment response, as well as a biological marker of cardio-metabolic changes. Similarly to Kaya et al, ${ }^{7}$ a recent prospective study also evaluated CIMT, ABI, additionally to flow-mediated dilation, mitral and tricuspidal annular plane systolic excursion, at 3 and 6 months after LSG. Correspondingly, authors concluded that all the analyzed parameters withstood a significant improvement despite a short time from surgery, validating LSG as a metabolic procedure to all effects. ${ }^{11}$

It would be worth investigating the influence of insulin resistance on the aforementioned atherosclerotic disease markers assessed by Kaya et al. ${ }^{7}$ In fact, insulin resistance is strictly associated with, and is a strong predictor of, CVD. A hyperinsulinemic state has been shown to promote the atherogenic process through several mechanisms, including activation of pro-inflammatory genes, increased lipogenesis, and proliferation of vascular smooth muscle cells. ${ }^{12}$ Furthermore, obesity is widely recognized as an insulin-resistant condition. ${ }^{13,14}$ Lack of evaluation of insulin resistance in the recruited obese patients in this study may hamper the possibility of correctly attributing the improvement of atherosclerotic vascular disease markers to the normalization of insulin sensitivity rather than to mere weight loss. Even though insulin resistance may improve consequently to the surgically-induced weight loss, a more indepth study of glucose tolerance and insulin resistance, using perhaps a euglycemic hyperinsulinemic clamp, might have allowed to reach more conclusive results.

In consideration of the fact that CVD remains a leading cause of morbidity and mortality, especially in the obese subpopulation, the identification of preclinical vascular markers would be of great value to potentially lead to early diagnosis and possibly to the prevention of such conditions. Indeed, APV represents a direct marker of arterial stiffness and has been shown to be altered in patients with atherosclerosis. ${ }^{15}$ It is of interest how Kaya et $\mathrm{al}^{7}$ found a significant increase in APV values only 6 months after LSG, indicating that this surgical bariatric procedure has the capacity to induce a profound improvement of the atherosclerotic vascular disease also in the short term, at least from an echocardiographic point of view. Despite this, further studies in the ambit of randomized controlled trials are warranted in order to better understand the effects of BMS on echocardiographic atherosclerotic vascular changes, trying to find actual causal factors responsible for these modifications which go beyond weight loss and that could be related to the resolution of comorbidities such as hypertension, dyslipidemia, insulin resistance, and diabetes.

\section{ARTICLE INFORMATION}

DISCLAIMER The opinions expressed by the author are not necessarily those of the journal editors, Polish Cardiac Society, or publisher.

CONFLICT OF INTEREST None declared.

OPEN ACCESS This is an Open Access article distributed under the terms of the Creative Commons Attribution-NonCommercial-NoDerivatives $4.0 \mathrm{In}$ ternational License (CC BY-NC-ND 4.0), allowing third parties to download articles and share them with others, provided the original work is properly cited, not changed in any way, distributed under the same license, and used for noncommercial purposes only. For commercial use, please contact the journal office at kardiologiapolska@ptkardio.pl.

How To CITE Casella G, Castagneto-Gissey L. Short-term weight-mediated effects of sleeve gastrectomy on echocardiographic surrogate markers of atherosclerotic vascular disease. Kardiol Pol. 2020; 78: 655-656. doi:10.33963/KP.15560

\section{REFERENCES}

1 World Health Organization. Obesity. Fact sheet 311, Updated March 2020. https://www.who.int/news-room/fact-sheets/detail/obesity-and-overweight. Accessed April 25, 2020.

2 Haslam DW, James WP. Obesity. Lancet. 2005; 366: 1197-1209.

3 Chang SH, Stoll CR, Song J, et al. The effectiveness and risks of bariatric surgery: an updated systematic review and meta-analysis, 2003-2012. JAMA Surg. 2014; 149: 275-287.

4 Kwok CS, Pradhan A, Khan MA, et al. Bariatric surgery and its impact on cardiovascular disease and mortality: a systematic review and meta-analysis. Int J Cardiol. 2014; 173: 20-28.

5 Switzer NJ, Prasad S, Debru E, et al. Sleeve gastrectomy and type 2 diabetes mellitus: a systematic review of long-term outcomes. Obes Surg. 2016; 26: 1616-1621.

6 Castagneto Gissey L, Casella Mariolo JR, Genco A, et al. 10-year follow-up after laparoscopic sleeve gastrectomy: outcomes in a monocentric series. Surg Obes Relat Dis. 2018; 14: 1480-1487.

7 Kaya BC, Elkan $\mathrm{H}$. The impact of weight loss after laparoscopic sleeve gastrectomy on early markers of atherosclerotic vascular disease: a prospective study. Kardiol Pol. 2020; 78: 674-680.

8 Tuluce K, Kara C, Tuluce SY, et al. Early reverse cardiac remodeling effect of laparoscopic sleeve gastrectomy. Obes Surg. 2017; 27: 364-375.

9 Sanches E, Timmermans M, Topal B, et al. Cardiac remodeling in obesity and after bariatric and metabolic surgery; is there a role for gastro-intestinal hormones? Expert Rev Cardiovasc Ther. 2019; 17: 771-790.

10 Cavarretta E, Casella G, Cali B, et al. Cardiac remodeling in obese patients after laparoscopic sleeve gastrectomy. World J Surg. 2013; 37: 565-572.

11 Tromba L, Tartaglia F, Carbotta S, et al. the role of sleeve gastrectomy in reducing cardiovascular risk. Obes Surg. 2017; 27: 1145-1151.

12 Di Pino A, DeFronzo RA. Insulin Resistance and atherosclerosis: implications for insulin-sensitizing agents. Endocr Rev. 2019; 40: 1447-1467.

13 Mingrone G, Castagneto-Gissey L. Type 2 diabetes mellitus in 2013: a central role of the gut in glucose homeostasis. Nat Rev Endocrinol. 2014; 10: 73-74.

14 DeFronzo RA. Insulin resistance, lipotoxicity, type 2 diabetes and atherosclerosis: the missing links. The Claude Bernard Lecture 2009. Diabetologia. 2010; 53 : 1270-1287.

15 Ghaderi F, Samim H, Keihanian F, Danesh Sani SA. The predictive role of aortic propagation velocity for coronary artery disease. BMC Cardiovasc Disord. 2018; 18: 121. 